\title{
Some Classes of Point Processes
}

\section{José Carlos S. de Miranda}

Universidade de São Paulo - Brazil

E-mail address: simon@ime.usp.br

\begin{abstract}
We define four classes of point processes which we call A, $\mathrm{B},{ }^{*} \mathrm{~A},{ }^{*} \mathrm{~B}$. Although we study point processes on $\mathbb{R}$, these classes are suitable for generalizations for point processes on $\mathbb{R}^{m}$ and other measure metric spaces. The main result is the equivalence of classes *A and ${ }^{*} \mathrm{~B}$ for point processes on $\mathbb{R}$. As a matter of fact, we prove that $A \varsubsetneqq B \varsubsetneqq * A=* B \varsubsetneqq S$, where $S$ is the class of simple processes. We also relate these classes and the class of Poisson processes.
\end{abstract}

\section{Introduction}

Point process intensity estimation is an important topic both for theoretical and practical purposes. The estimation of the intensity may be done via wavelets (see [1], [2], [3], [4], [9], [10] and [11]). In practice, it is common not to know if the intensity of a process belongs to some class of functions or not. Thus it is important to have methods that can deal with as wide as possible classes of point processes. In what follows, we will define classes of point processes that are suitable for wavelet estimation of their intensity. These are very wide classes. Particularly, the conditions to be fulfilled by a point process to belong to ${ }^{*} \mathrm{~B}$ are really mild.

This article is organized as follows. In section 2 we present some background and notation for point processes. In section 3 the classes are defined. Section 4 is devoted to establish the interrelationship between classes and in section 5 we study the special case of Poisson processes. In section 6 we present a short conclusion.

Key words: Classes of point processes, hypotheses A, B, *A and ${ }^{*} \mathrm{~B}$, infinitesimal, intensity, non-internally correlated, point process, Poisson process, product density.

The author thanks OLSJC . 


\section{Point Processes Background and Notation}

2.1. Notation. We will work with Lebesgue measurable functions, $h$ : $\mathbb{R}^{m} \rightarrow \mathbb{R}$ that are bounded over bounded $\mathbb{R}^{m}$-intervals or, equivalently, that are Lebesgue integrable and bounded over bounded $\mathbb{R}^{m}$ - intervals. We will call this class of functions $\mathcal{L}^{m}$. We will denote by $\overline{\mathcal{L}}^{m}$ the class of Lebesgue integrable functions over bounded $\mathbb{R}^{m}$-intervals. The class of Riemann integrable functions $h: \mathbb{R}^{m} \rightarrow \mathbb{R}$ over bounded $\mathbb{R}^{m}$-intervals will be denoted by $\mathcal{R}^{m}$. Since all Riemann integrable functions over bounded intervals are bounded over these intervals, we have $\mathcal{R}^{m} \subset \mathcal{L}^{m}$.

The Lebesgue measure on $\mathbb{R}^{m}$ will be simply denoted by $\ell$ independently of the dimension $m$. Whenever it is necessary or to emphasize dimension, we will write $\ell_{m}$. The $\sigma$-algebra of Lebesgue measurable sets in $\mathbb{R}^{m}$ is denoted by $\Lambda_{\mathbb{R}^{m}}$ and $\mathcal{B}_{\mathbb{R}^{m}}$ stands for the $\sigma$-algebra of Borel sets. Functions that only differ over zero measure subsets of their common domain or of a common extension of their domains are naturally considered identical. All functions are considered to be measurable.

We denote by $N(A)$ the number of events that occur in $A \subset \mathbb{R}$. If $A=(\alpha, \beta]$, we write $N(\alpha, \beta]$ instead of $N((\alpha, \beta])$. We also denote by $N$ the integer valued function defined by the equalities $N(t)=N(0, t]$, if $t>$ $0, N(0)=0$ and $N(t)=-N(t, 0]$ if $t<0$. Clearly $N(\alpha, \beta]=N(\beta)-N(\alpha)$. Let $\left\{\cdots, \tau_{-2} \leq \tau_{-1} \leq \tau_{0} \leq 0<\tau_{1} \leq \tau_{2} \leq \cdots\right\}$ denote the times at which the events occur. Then $N(t)=n$, if and only if $\tau_{n} \leq t<\tau_{n+1}$.

Provided that probabilities of the form

$$
P\left(N\left(\alpha_{1}, \beta_{1}\right]=n_{1}, \ldots, N\left(\alpha_{k}, \beta_{k}\right]=n_{k}\right)
$$

are defined and consistent, for all $k \in \mathbb{N}^{*}=\{1,2, \ldots\}$, and all $n_{1}, \ldots, n_{k}$ non-negative integers, we can define an appropriate probability space $(\Omega, \mathcal{A}, P)$, such that there exists a measurable mapping from this space to $\left(\mathbb{R}^{\mathbb{Z}}, \mathcal{B}_{\mathbb{R}^{\mathbb{Z}}}\right)$, defining then a stochastic point process that will also be called $N$.

2.2. Intensity and Product Density. Suppose that there exists a positive real number $\delta$ and a constant $K_{\delta}>0$ such that for all intervals $\Delta \subset \mathbb{R}$ with length $|\Delta|<\delta$, all integers $n>1$ and all $t \in \mathbb{R}$, not only the relation

$$
P\{N(\Delta)=n\} \leq K_{\delta}|\Delta|^{n}
$$

holds, but also the limit

$$
\lim _{|\Delta| \rightarrow 0, t \in \Delta} \frac{1}{|\Delta|} P\{N(\Delta)=1\}=p_{N}(t)
$$


exists uniformly in $t$. Inequality (1) implies that

$$
P\{N(\Delta)>1\} \leq K_{\delta}\left(\sum_{j \geq 2}|\Delta|^{j}\right)=O\left(|\Delta|^{2}\right) .
$$

Note that if inequality (1) were valid for $n=1$ then we would have $P\{N(\Delta)=1\} /|\Delta| \leq K_{\delta}$ and hence, if it would exist, $p_{N}(t)$ would be a bounded function on $\mathbb{R}$. Also observe that (2) implies that $\forall x \in \mathbb{R}$, $P\{N(\{x\})=1\}=0$, otherwise there would exist $t \in \mathbb{R}$ for which the limit $p_{N}(t)$ would be infinite.

Due to uniformity, relation (2) is equivalent to

$$
P\{N(\Delta)=1\}=p_{N}(t)|\Delta|+o_{t, \Delta}(|\Delta|),
$$

for an infinitesimal $o_{t, \Delta}(z)$ with the following properties:

$\forall \varepsilon>0, \exists \delta>0, \forall t \in \mathbb{R}, \forall \Delta \subset \mathbb{R}, t \in \Delta,(0<|\Delta|<\delta) \rightarrow\left|o_{t, \Delta}(|\Delta|)\right| \leq \frac{\varepsilon}{2}|\Delta|$ and $o_{t, \Delta}(0)=0$,

that is, $\forall \varepsilon>0, \exists \delta>0,(0<z<\delta) \rightarrow \sup _{\substack{t \in \mathbb{R}, \Delta \subset \mathbb{R} \\ t \in \Delta,|\Delta|=z}}\left|o_{t, \Delta}(z)\right| \leq \frac{\varepsilon}{2} z<\varepsilon z$ and $o_{t, \Delta}(0)=0$.

The quantity $\sup _{\substack{t \in \mathbb{R}, \Delta \subset \mathbb{R} \\ t \in \Delta,|\Delta|=z}}\left|o_{t, \Delta}(z)\right|=o(z)$ is a non-negative infinitesimal independent of $t$ and $\Delta$. In this sense, we also write $\left|o_{t, \Delta}(|\Delta|)\right| \leq o(|\Delta|)$.

For the ease of notation, we will write $o_{t}$ instead of $o_{t, \Delta}$.

We say that $p_{N}(t)$ is the intensity of occurrence of events at time $t$, more precisely, of single occurrence of events at $t$.

Suppose now that there exists a positive real number $\delta$ and a constant $k_{\delta, m}$ such that for all intervals $\Delta_{1}, \ldots, \Delta_{m}$ of the real line with lengths $0<\left|\Delta_{i}\right|<\delta, 1 \leq i \leq m$, all integers $n_{i} \geq 1$ and all vectors $\left(t_{1}, \ldots, t_{n}\right) \in \mathbb{R}^{m}$ with $t_{i} \neq t_{j}$ for $i \neq j, 1 \leq i \leq m, 1 \leq j \leq m$, both properties below are valid:

if $\left(n_{1}, \ldots, n_{m}\right) \neq(1, \ldots, 1)$ then $P\left\{N\left(\Delta_{i}\right)=n_{i}, 1 \leq i \leq m\right\} \leq k_{\delta, m} \prod_{i=1}^{m}\left|\Delta_{i}\right|^{n_{i}}$

and for $|\Delta|:=\left(\left|\Delta_{1}\right|, \ldots,\left|\Delta_{m}\right|\right) \in\left(\mathbb{R}_{+}^{*}\right)^{m}, t_{i} \in \Delta_{i}, 1 \leq i \leq m$, there exists the limit

$$
\lim _{|\Delta| \rightarrow 0} \frac{1}{\prod_{i=1}^{m}\left|\Delta_{i}\right|} P\left\{N\left(\Delta_{i}\right)=1,1 \leq i \leq m\right\}=p_{m}\left(t_{1}, \ldots, t_{m}\right) .
$$


The limit above measures the intensity of the joint occurrence of events in the distinct times $t_{1}, \ldots, t_{m}$. We might call it the joint intensity. Since under the relations (3) and (4) it is also valid that $\lim _{|\Delta| \rightarrow 0} \frac{1}{\prod_{i=1}^{m}\left|\Delta_{i}\right|} E\left\{\prod_{i=1}^{m} N\left(\Delta_{i}\right)\right\}$ $=p_{m}\left(t_{1}, \ldots, t_{m}\right), p_{m}$ is called product density of order $m$. Relation (4) implies that

$$
P\left\{N\left(\Delta_{i}\right)=1,1 \leq i \leq m\right\}=p_{m}\left(t_{1}, \ldots, t_{m}\right) \prod_{i=1}^{m}\left|\Delta_{i}\right|+o_{t, \prod_{i=1}^{m} \Delta_{i}}(|\Delta|)
$$

for $o_{t, \prod_{i=1}^{m} \Delta_{i}}(|\Delta|)$ an infinitesimal such that $\frac{o_{t, \prod_{i=1}^{m} \Delta_{i}}(|\Delta|)}{\prod_{i=1}^{m}\left|\Delta_{i}\right|} \rightarrow 0$ when $\Delta \rightarrow$ 0.

Again, for the ease of notation, we write $o_{t}$ instead of $o t, \prod_{i=1}^{m} \Delta_{i}$.

We can also define cumulants for $N(t)$; and in particular, we define the limit covariance, for $u \neq v$, by

$$
q_{2}(u, v)=\lim _{|\Delta| \rightarrow 0} \frac{\operatorname{Cov}(N, N)\left(\Delta_{1} \times \Delta_{2}\right)}{\left|\Delta_{1}\right|\left|\Delta_{2}\right|} .
$$

Whenever $p_{2}(u, v), p_{1}(u)$ and $p_{2}(v)$ exist, we write

$$
\begin{aligned}
q_{2}(u, v) & =\lim _{|\Delta| \rightarrow 0} \frac{\operatorname{Cov}(N, N)\left(\Delta_{1} \times \Delta_{2}\right)}{\left|\Delta_{1}\right|\left|\Delta_{2}\right|} \\
& =\lim _{|\Delta| \rightarrow 0} \frac{E\left(N\left(\Delta_{1}\right) N\left(\Delta_{2}\right)\right)}{\left|\Delta_{1}\right|\left|\Delta_{2}\right|}-\lim _{|\Delta| \rightarrow 0} \frac{E\left(N\left(\Delta_{1}\right)\right)}{\left|\Delta_{1}\right|} \frac{E\left(N\left(\Delta_{2}\right)\right)}{\left|\Delta_{2}\right|} \\
& =p_{2}(u, v)-p_{1}(u) p_{2}(v) .
\end{aligned}
$$

The following proposition and theorem will be useful in section 4 .

Proposition 2.1. Under conditions (1) and (2), we have

$$
\begin{aligned}
P\{N(\Delta)=1\} \leq E\{N(\Delta)\} & \leq P\{N(\Delta)=1\}+O\left(|\Delta|^{2}\right), \\
P\{N(\Delta)=1\}-A \leq \operatorname{Var}\{N(\Delta)\} & \leq P\{N(\Delta)=1\}+B,
\end{aligned}
$$

where $A$ and $B$ are $O\left(|\Delta|^{2}\right)$ whenever $\sup _{t \in \Delta} p_{N}(t)$ is finite.

Therefore we can write 


$$
E\{N(\Delta)\}=p_{N}(t)|\Delta|+o_{t}(|\Delta|)
$$

and

$$
\operatorname{Var}\{N(\Delta)\}=p_{N}(t)|\Delta|+o_{t}(|\Delta|)
$$

These infinitesimals $o_{t}=o_{t, \Delta}$ may depend on $t$ and $\Delta$ but their absolute values are bounded by other $o$ 's which are independent of $t$.

Theorem 2.1. Let $\mathcal{E}^{m}=\left\{x=\left(x_{1}, \ldots, x_{m}\right) \in \mathbb{R}^{m} \mid x_{i}=x_{j}\right.$ for some pair $i, j, i \neq j\}, \varphi$ an $E\left(\prod_{i=1}^{m} \mathrm{~d} N\left(t_{i}\right)\right)$-integrable function on $\mathbb{R}^{m} \backslash \mathcal{E}^{m}, p_{m}$ the $m$-th order product density and $p_{1}=p_{N}$ the intensity function of a point process $N$ that satisfies (3) and (4). Then, if $p_{m} \in \overline{\mathcal{L}}^{m}, m \geq 1$, we have

$$
\int_{\mathbb{R}^{m} \backslash \mathcal{E}^{m}} \varphi E\left(\prod_{i=1}^{m} \mathrm{~d} N\left(t_{i}\right)\right)=\int_{\mathbb{R}^{m} \backslash \mathcal{E}^{m}} \varphi p_{m} \prod_{i=1}^{m} \mathrm{~d} t_{i} .
$$
$\mathbb{R}^{2}$.

Clearly, $\mathcal{E}^{1}=\emptyset$ and $\mathcal{E}^{2}=D=\left\{(x, x) \in \mathbb{R}^{2} \mid x \in \mathbb{R}\right\}$ is the diagonal set of

Proofs and further results can be found in [4] and [9].

\section{Definition of Classes}

Definition 1. We will say that a point process $N$ satisfies

hypothesis B: when $N$ satisfies relations (1) and (2) and $p_{1}=p_{N} \in \overline{\mathcal{L}}^{1}$. Since (1), (2) and $p_{1} \in \overline{\mathcal{L}}^{1}$ imply $p_{1} \in C(\mathbb{R}, \mathbb{R}) \subset \mathcal{R}^{1} \subset \mathcal{L}^{1} \subset \overline{\mathcal{L}}^{1}$, hypothesis $B$ is equivalent to (1), (2) and $p_{1}$ continuous (see [4] and [5]).

hypothesis A: when $N$ satisfies hypothesis $B$ and also relations (3), (4) for $m=2$ and $p_{2} \in \overline{\mathcal{L}}^{2}, p_{2}$ essentially bounded over bounded intervals.

The classes $A$ and $B$ are defined by $N \in B$ if and only if $N$ satisfies hypothesis $B$ and $N \in A$ if and only if $N$ satisfies hypothesis $A$.

Next we define two wider classes of point process.

Definition 2. A point process $N$ satisfies hypotheses ${ }^{*} B$ when not only its expectation measure is absolutely continuous with respect to the Lebesgue measure, $E N \ll \ell$, that is, when there exists $\mathrm{d} E N / \mathrm{d} \ell \in \overline{\mathcal{L}}^{1}$, but also the following relation holds: $\forall t \in \mathbb{R} \forall \Delta \subset \mathbb{R}, \Delta$ interval, $t \in \Delta, E N(\Delta)=$ $P\{N(\Delta)=1\}+o_{t, \Delta}(|\Delta|)$. 

${ }^{*} B$.

The class ${ }^{*} B$ is defined by $N \in{ }^{*} B$ if and only if $N$ satisfies hypothesis

Theorem 3.1. Let $N$ be a point process that satisfies hypothesis ${ }^{*} B$. Then the intensity defining limit $p_{N}$ exists and $\mathrm{d} E N / \mathrm{d} \ell=p_{N}$ a.e. $[\ell]$.

Proof: For all $t \in \mathbb{R}$, we compute the intensity defining limit $p_{N}(t)$ :

$$
p_{N}(t)=\lim _{\substack{t \in \Delta \\|\Delta| \rightarrow 0}} \frac{P\{N(\Delta)=1\}}{|\Delta|}=\lim _{\substack{t \in \Delta \\|\Delta| \rightarrow 0}} \frac{E N(\Delta)-o_{t}(|\Delta|)}{|\Delta|}=\lim _{\substack{t \in \Delta \\|\Delta| \rightarrow 0}} \frac{E N(\Delta)}{|\Delta|},
$$

Let $f=\frac{\mathrm{d} E N}{\mathrm{~d} \ell}, \varphi(x)=\int_{c}^{x} f(y) \mathrm{d} y, \Delta=|a, b|, a<b, h_{1}=b-t$ and $h_{2}=t-a$. Thus,

$$
p_{N}(t)=\lim _{\substack{h_{1} \rightarrow 0 \\ h_{2} \rightarrow 0}} \frac{\varphi\left(t+h_{1}\right)-\varphi\left(t-h_{2}\right)}{h_{1}+h_{2}} .
$$

Now,

$$
\begin{gathered}
\frac{\varphi\left(t+h_{1}\right)-\varphi\left(t-h_{2}\right)}{h_{1}+h_{2}}= \\
=\frac{\varphi\left(t+h_{1}\right)-\varphi(t)}{h_{1}} \frac{h_{1}}{h_{1}+h_{2}}+\frac{\varphi\left(t-h_{2}\right)-\varphi(t)}{-h_{2}} \frac{h_{2}}{h_{1}+h_{2}} \\
=\left(f(t)+o_{t}\left(h_{1}\right)\right) \frac{h_{1}}{h_{1}+h_{2}}+\left(f(t)+o_{t}\left(-h_{2}\right)\right) \frac{h_{2}}{h_{1}+h_{2}} \\
=f(t)+\left(o_{t}\left(h_{1}\right) \frac{h_{1}}{h_{1}+h_{2}}+o_{t}\left(-h_{2}\right) \frac{h_{2}}{h_{1}+h_{2}}\right),
\end{gathered}
$$

where, by Lebesgue's differentiation theorem, $o_{t}$ is an infinitesimal a.e. $[\ell]$ (this means that the set of $t$ 's such that $o_{t}$ is not an infinitesimal has zero Lebesgue measure).

Since $0 \leq \frac{h_{1}}{h_{1}+h_{2}} \leq 1$ and $0 \leq \frac{h_{2}}{h_{1}+h_{2}} \leq 1$, we have

$$
\lim _{\substack{h_{1} \rightarrow 0 \\ h_{2} \rightarrow 0}} \frac{\varphi\left(t+h_{1}\right)-\varphi\left(t-h_{2}\right)}{h_{1}+h_{2}}=f(t)+0 \text { a.e. }[\ell] \text {. }
$$

Thus, $p_{N}(t)=\frac{\mathrm{d} E N}{\mathrm{~d} \ell}$ a.e. $[\ell]$.

Definition 3. A point process $N$ satisfies hypotheses ${ }^{*} A$ when it is under hypotheses ${ }^{*} B$ and the equality

$$
E(N \times N)(A \cap D)=E N \pi_{1}(A \cap D)
$$


holds for all $A \in \Lambda_{\mathbb{R}^{2}}$, where $D$ is the diagonal set of $\mathbb{R}^{2}$ and $\pi_{1}$ is the first canonical projection.

The class ${ }^{*} A$ is defined by $N \in{ }^{*} A$ if and only if $N$ satisfies hypothesis ${ }^{*} A$.

We observe that this condition is equivalent to say that the measure $E(N \times N)$ restricted to diagonal, $\left.E(N \times N)\right|_{D}: \Lambda_{D} \rightarrow \mathbb{R}$, is the induced measure over the diagonal by the measure $E N$ on the real line through $\pi_{1}$, that is, $\left.E(N \times N)\right|_{D}=E N \pi_{1}$.

Definition 4. A point process is called non-internally correlated if and only if for all $A$ and $B$ disjoint Lebesgue measurable sets we have $\operatorname{Cov}(N(A), N(B))=0$. [8].

For non trivial examples of non internally correlated point processes, see

The following example shows the existence of point processes outside $* B$. It also shows that $E N \ll \ell$ does not imply $\forall t \in \mathbb{R} \forall \Delta \subset \mathbb{R}, \Delta$ interval, $t \in \Delta, E N(\Delta)=P\{N(\Delta)=1\}+o_{t, \Delta}(|\Delta|)$.

Example: Let $N$ be a Poisson point process on $\mathbb{R}$ with intensity $\lambda$. Let $M=2 * N$. Then, $E M \ll \ell$ and for all $\Delta, E M(\Delta)=2 \lambda|\Delta|$ and $P(M(\Delta)=$ $1)=0$. Thus $E M(\Delta)=2 \lambda|\Delta| \neq P\{N(\Delta)=1\}+o_{t, \Delta}(|\Delta|)=0+o_{t, \Delta}(|\Delta|)$ and $M \notin * B$.

\section{Interrelationships Between Classes}

Theorem 4.1. If $N$ satisfies hypothesis $B$ then $N$ is also under hypotheses ${ }^{*} B$.

Proof: Since $N$ satisfies hypothesis B, we not only have $p_{N} \in \overline{\mathcal{L}^{1}}$ but also, by Theorem $2.1, p_{N}=\mathrm{d} E N / \mathrm{d} \ell$ a.e. $[\ell]$. Thus $\mathrm{d} E N / \mathrm{d} \ell \in \overline{\mathcal{L}^{1}}$. Now, since proposition (2.1) hypothesis is satisfied by point processes under hypothesis $\mathrm{B}$ we have

$$
P\{N(\Delta)=1\} \leq E\{N(\Delta)\} \leq P\{N(\Delta)=1\}+O\left(|\Delta|^{2}\right),
$$

from which $E\{N(\Delta)\}=p_{N}(t)|\Delta|+o_{t, \Delta}(|\Delta|)$

Theorem 4.2. If $N$ satisfies hypothesis $A$ then $N$ is also under hypotheses ${ }^{*} A$.

Proof: Since $N$ satisfies hypothesis A, it also satisfies B which implies $N$ satisfies ${ }^{*} \mathrm{~B}$. 
For more details on the following part of this proof, see [4], [5] and [6].

Denoting by $d u$ not only the number $d u$ but also the interval $[u, u+d u)$, we write

$$
\begin{gathered}
E(N \times N)(d u \times d u)=E(N[u, u+d u) N[u, u+d u)) \\
P\{N[u, u+d u)=1\} \leq E\left\{N[u, u+d u)^{2}\right\} \leq \\
\leq P\{N[u, u+d u)=1\}+\sum_{j \geq 2} j^{2} P\{N[u, u+d u)=j\} .
\end{gathered}
$$

Thus, $E\left\{N[u, u+d u)^{2}\right\}=P\{N[u, u+d u)=1\}+o_{u}^{\prime}(d u)=p_{N}(u) d u+$ $o_{u}(d u)$.

$E(N \times N)((d u \times d u) \backslash D)=\int_{u}^{u+d u} \int_{u}^{u+d u} p_{2} d \ell<M(d u)^{2}$, since, as $N$ satisfies hypothesis $\mathrm{A}$, there exists $M>0$, such that $p_{2}(x, y)<M$ a.e. [ $\left.\ell\right]$ in $d u \times d u$.

Let $\left.E(N \times N)\right|_{D}(A)=E(N \times N)(A \cap D)$, for all $A \in \Lambda_{\mathbb{R}^{2}}$.

Thus, $p_{N}(u) d u+o_{u}(d u)-M(d u)^{2} \leq\left. E(N \times N)\right|_{D}(d u \times d u) \leq p_{N}(u) d u+$ $o_{u}(d u)$ and, consequently, $\left.E(N \times N)\right|_{D}(d u \times d u)=p_{N}(u) d u+o_{u}^{\prime \prime}(d u)$.

Neglecting higher order infinitesimals, we have

$$
\left.\int_{A \cap D} d E(N \times N)\right|_{D}=\int_{\pi_{1}(A \cap D)} p_{N}(u) d u=\int_{\pi_{1}(A \cap D)} \frac{d E N}{d \ell} d \ell
$$
i.e.,

$$
E(N \times N)(A \cap D)=E N \pi_{1}(A \cap D)
$$

Definition 5. A point process is called strictly simple if and only if $\forall \omega \in \Omega$ $\forall t \in \mathbb{R} N_{\omega}(\{t\}) \in\{0,1\}$.

That is, $N$ is strictly simple when there are no simultaneous occurrences of events.

Theorem 4.3. If $N$ is a strictly simple point process under hypothesis ${ }^{*} B$ then $N$ also satisfies hypothesis ${ }^{*}$ A.

Proof: Take $A \in \Lambda_{\mathbb{R}}$ bounded and let $\Omega_{A}^{\prime}=\left\{\omega \in \Omega \mid N_{\omega}(A)=\infty\right\}$. As $\mathrm{d} E N / \mathrm{d} \ell \in \overline{\mathcal{L}}^{1}$ we have $\int_{A}(\mathrm{~d} E N / \mathrm{d} \ell) \mathrm{d} \ell=E N(A)<\infty$. Thus $P\left(\Omega_{A}^{\prime}\right)=0$, otherwise we would have $E N(A)=\int_{\Omega \backslash \Omega_{A}^{\prime}} N_{\omega}(A) \mathrm{d} P+\int_{\Omega_{A}^{\prime}} N_{\omega}(A) \mathrm{d} P=\infty$.

Now, for all bounded $A \in \Lambda_{\mathbb{R}}$ and $\forall \omega \in \Omega \backslash \Omega_{A}^{\prime}$ we can write the measure $\left.N_{\omega}\right|_{A}$ as a counting measure $\left.N_{\omega}\right|_{A}=\sum_{j \in J_{A}(\omega)} n_{j}(\omega) \delta_{x_{j}(\omega)}, J_{A}(\omega)$ finite 
set, $\forall j \in J_{A}(\omega), x_{j}(\omega) \in A$ and $\left.N_{\omega}\right|_{A}(A)=\sum_{j \in J_{A}(\omega)} n_{j}(\omega) \delta_{x_{j}(\omega)}(A)=$ $\sum_{j \in J_{A}(\omega)} n_{j}(\omega) \in \mathbb{N}$. In this way, $\forall \omega \in \Omega \backslash \Omega_{A}^{\prime}$ we have

$$
\begin{gathered}
\left.N_{\omega}\right|_{A} \times\left. N_{\omega}\right|_{A}=\sum_{j_{1} \in J_{A}(\omega)} n_{j_{1}}(\omega) \delta_{x_{j_{1}}(\omega)} \times \sum_{j_{2} \in J_{A}(\omega)} n_{j_{2}}(\omega) \delta_{x_{j_{2}}(\omega)}= \\
=\sum_{\left(j_{1}, j_{2}\right) \in J_{A}^{2}(\omega)} n_{j_{1}}(\omega) n_{j_{2}}(\omega) \delta_{x_{j_{1}}(\omega)} \times \delta_{x_{j_{2}}(\omega)}= \\
=\sum_{\left(j_{1}, j_{2}\right) \in J_{A}^{2}(\omega)} n_{j_{1}}(\omega) n_{j_{2}}(\omega) \delta_{\left(x_{j_{1}}(\omega), x_{j_{2}}(\omega)\right)} .
\end{gathered}
$$

Thus,

$$
\begin{gathered}
N_{\omega} \times\left. N_{\omega}\right|_{A \times A}((A \times A) \cap D)= \\
=\sum_{\left(j_{1}, j_{2}\right) \in J_{A}^{2}(\omega)} n_{j_{1}}(\omega) n_{j_{2}}(\omega) \delta_{\left(x_{j_{1}}(\omega), x_{j_{2}}(\omega)\right)}(\{(x, x) \mid x \in A\})= \\
=\sum_{k \in J_{A}(\omega)} n_{k}^{2}(\omega) \delta_{\left(x_{k}(\omega), x_{k}(\omega)\right)}(\{(x, x) \mid x \in A\})=\sum_{k \in J_{A}(\omega)} n_{k}^{2}(\omega) \delta_{x_{k}(\omega)}(A) .
\end{gathered}
$$

As $N$ is simple, for all $k, n_{k}^{2}(\omega)=n_{k}(\omega)=1$; we have $N_{\omega} \times\left. N_{\omega}\right|_{A \times A}((A \times$ $A) \cap D)=\# J_{A}(\omega)=\left.N_{\omega}\right|_{A}(A)$.

In this way, we have obtained $\forall A \in \Lambda_{\mathbb{R}}, A$ bounded, $\forall \omega \in \Omega \backslash \Omega_{A}^{\prime}$, $P\left(\Omega_{A}^{\prime}\right)=0,\left(N_{\omega} \times N_{\omega}\right)((A \times A) \cap D)=N_{\omega}(A)$.

Thus, $E(N \times N)((A \times A) \cap D)=\int_{\Omega \backslash \Omega_{A}^{\prime}}\left(N_{\omega} \times N_{\omega}\right)((A \times A) \cap D) \mathrm{d} P(\omega)=$ $\int_{\Omega \backslash \Omega_{A}^{\prime}} N_{\omega}(A) \mathrm{d} P(\omega)=E N(A)$. That is $E(N \times N)((A \times A) \cap D)=E N(A)$.

Now, let $C \in \Lambda_{\mathbb{R}^{2}}$. If $C$ is bounded $E(N \times N)(C \cap D)=E(N \times$ $N)\left(\left(\pi_{1}(C \cap D) \times \pi_{1}(C \cap D)\right) \cap D\right)=E N\left(\pi_{1}(C \cap D)\right)$. If $C$ is not bounded, let $I_{z}^{D}=[z, z+1)^{2} \cap D$, so that $C \cap I_{z}^{D}$ is bounded. We have $E(N \times N)(C \cap D)=$ $\sum_{z \in \mathbb{Z}} E(N \times N)\left(C \cap D \cap I_{z}^{D}\right)=\sum_{z \in \mathbb{Z}} E N\left(\pi_{1}\left(C \cap D \cap I_{z}^{D}\right)\right)=E N\left(\pi_{1}(C \cap D)\right)$. This completes the proof.

Definition 6. A point process is called simple if and only if $P\{\omega \in \Omega \mid \exists t \in$ $\left.\mathbb{R} N_{\omega}(\{t\})>1\right\}=0$.

Lemma 4.1. Let a point process $N$ defined on $(\Omega, \mathcal{A}, P)$ be under hypothesis ${ }^{*} B$. Then, if $\Omega_{1} \subset \Omega$ is such that $P\left(\Omega_{1}\right)=1$, the point process $N \mid \Omega_{1}$, restriction of $N$ to $\Omega_{1}$, is still under hypothesis ${ }^{*} B$.

Proof: We denote $\left.N\right|_{\Omega_{1}}$ the restriction of the function $N$ to $\Omega_{1}$ subset of its domain $\Omega$. This restriction naturally inherits a point process structure. 
It suffices to take $\mathcal{A}_{1}=\mathcal{A} \cap \Omega_{1}=\left\{B \cap \Omega_{1} \mid B \in \mathcal{A}\right\}$ and $P_{1}=\left.P\right|_{\mathcal{A}_{1}}: \mathcal{A}_{1} \rightarrow$ $[0,1]$.

Now, for all $A \in \Lambda_{\mathbb{R}}$ we have

$$
\begin{array}{r}
\left.E N\right|_{\Omega_{1}}(A)=\left.\int_{\Omega_{1}} N\right|_{\Omega_{1}}(A) d P_{1}=\left.\int_{\Omega_{1}} N\right|_{\Omega_{1}}(A) d P= \\
=\left.\int_{\Omega_{1}} N\right|_{\Omega_{1}}(A) d P+\int_{\Omega \backslash \Omega_{1}} N_{\omega}(A) d P-\int_{\Omega \backslash \Omega_{1}} N_{\omega}(A) d P= \\
=\int_{\Omega} N_{\omega}(A) d P-\int_{\Omega \backslash \Omega_{1}} N_{\omega}(A) d P=E N(A)
\end{array}
$$

since $P\left(\Omega \backslash \Omega_{1}\right)=0$. So $\left.E N\right|_{\Omega_{1}}=E N$.

In this way we have $\left.E N\right|_{\Omega_{1}}=E N \ll \ell$ and $\left.E N\right|_{\Omega_{1}}(\Delta)=E N(\Delta)=$ $P\{N(\Delta)=1\}+o_{t, \Delta}(|\Delta|)=P\left\{\omega \in \Omega_{1} \mid N(\Delta)=1\right\}+P\left\{\omega \in \Omega \backslash \Omega_{1} \mid N(\Delta)=\right.$ $1\}+o_{t, \Delta}(|\Delta|)=P\left\{\left.N\right|_{\Omega_{1}}(\Delta)=1\right\}+o_{t, \Delta}(|\Delta|)$.

Corollary 4.1. If $N$ is a simple point process that satisfies hypothesis ${ }^{*} B$ then it also satisfies hypothesis ${ }^{*} A$.

Proof: Let $\Omega=\Omega_{1} \cup \Omega_{2}$ where

$$
\begin{aligned}
& \Omega_{1}=\{\omega \in \Omega \mid \nexists t \in \mathbb{R} N(\{t\})>1\} \\
& \Omega_{2}=\{\omega \in \Omega \mid \exists t \in \mathbb{R} N(\{t\})>1\} .
\end{aligned}
$$

Since $N$ is simple we have $P\left(\Omega_{1}\right)=1$ and $P\left(\Omega_{2}\right)=0$. Furthermore $\left.N\right|_{\Omega_{1}}$ is a strictly simple process under ${ }^{*} \mathrm{~B}$ and this implies that $\left.N\right|_{\Omega_{1}}$ satisfies hypothesis *A. So we write:

$$
\begin{aligned}
E(N \times N)(A \cap D) & =E\left((N \times N)(A \cap D) \mid \omega \in \Omega_{1}\right) P\left\{\omega \in \Omega_{1}\right\} \\
& +E\left((N \times N)(A \cap D) \mid \omega \in \Omega_{2}\right) P\left\{\omega \in \Omega_{2}\right\} \\
& =E\left(N \pi_{1}(A \cap D) \mid \omega \in \Omega_{1}\right) \\
& =E\left(N \pi_{1}(A \cap D) \mid \omega \in \Omega_{1}\right) P\left(\Omega_{1}\right) \\
& +E\left(N \pi_{1}(A \cap D) \mid \omega \in \Omega_{2}\right) P\left(\Omega_{2}\right) \\
& =E N \pi_{1}(A \cap D) .
\end{aligned}
$$

Lemma 4.2. If $N$ satisfies relation (5) then it is simple. 
Proof: Let for all $n \in \mathbb{N}^{*}$ for all $i, 0 \leq i \leq n-1, \Delta_{i}=[i / n,(i+1) / n)$. Then, for all $n \in \mathbb{N}^{*}$

$$
\begin{aligned}
P\{\omega \in \Omega \mid \exists t & \in[0,1) N(\{t\})>1\} \leq \\
& \leq P\left\{\omega \in \Omega \mid \exists i, 0 \leq i \leq n-1, N\left(\Delta_{i}\right)>1\right\} \\
& \leq \sum_{i=1}^{n} P\left\{N\left(\Delta_{i}\right)>1\right\} \leq \sum_{i=1}^{n}\left(\sum_{j \geq 2} K_{\delta}\left|\Delta_{i}\right|^{j}\right) \\
& =\sum_{i=1}^{n} K_{\delta}\left|\Delta_{i}\right|^{2}\left(\frac{1}{1-\left|\Delta_{i}\right|}\right)=K_{\delta} \frac{1}{1-1 / n} \frac{1}{n}\left(\sum_{i=1}^{n} \frac{1}{n}\right)=\frac{K_{\delta}}{n-1} .
\end{aligned}
$$

Letting $n \rightarrow \infty$ we have $0 \leq P\{\omega \in \Omega \mid \exists t \in[0,1) N(\{t\})>1\} \leq 0$.

Repeating this argument for the intervals $[m, m+1), m \in \mathbb{Z}$ we write:

$$
\begin{aligned}
0 & \leq P\{\omega \in \Omega \mid \exists t \in \mathbb{R} N(\{t\})>1\} \\
& \leq \sum_{m \in \mathbb{Z}} P\{\omega \in \Omega \mid \exists t \in[m, m+1) N(\{t\})>1\} \leq \sum_{m \in \mathbb{Z}} 0=0 .
\end{aligned}
$$

Theorem 4.4. If $N$ satisfies hypothesis $B$ then it is simple.

Proof: Direct consequence of Lemma 4.2.

Corollary 4.2. If $N$ satisfies hypothesis $B$, then $N$ satisfies hypothesis ${ }^{*} A$.

Proof: Since $N$ satisfies hypothesis $\mathrm{B}$, it also satisfies hypothesis ${ }^{*} \mathrm{~B}$. Now, by Theorem 4.4, $N$ is simple and the conclusion follows by Corollary 4.1.

Theorem 4.5. If $N$ is a point process under hypothesis * $A$ then it is simple.

Proof: Suppose it is not so. That is, $N$ is under hypothesis $*$ A and it is not simple. Then, $\Omega_{2}=\{\omega \in \Omega \mid \exists t \in \mathbb{R} N(\{t\})>1\}$ is such that $P\left(\Omega_{2}\right)>0$. Let $\Omega_{2, m}=\{\omega \in \Omega \mid \exists t \in[m, m+1) N(\{t\})>1\}$. In this way $\Omega_{2} \subset \bigcup_{m \in \mathbb{Z}} \Omega_{2, m}$ and there is an $m_{1} \in \mathbb{Z}$ such that $P\left(\Omega_{2, m_{1}}\right)>0$, otherwise we would have $P\left(\Omega_{2}\right) \leq \sum_{m \in \mathbb{Z}} P\left(\Omega_{2, m}\right)=0$. Now, since $N$ is under hypothesis *A it is under hypothesis ${ }^{*} \mathrm{~B}$, consequently, $p_{N} \in \overline{\mathcal{L}}^{1}$ and the set $\Omega^{\prime \prime}$ such that if $\omega \in \Omega^{\prime \prime}$ then $N\left(\left[m_{1}, m_{1}+1\right)\right)=\infty$ has zero probability. Let 
$\Omega^{\prime}=\Omega \backslash \Omega^{\prime \prime}$. Let $X$ be the random variable, $X=(N \times N)\left(\left[m_{1}, m_{1}+1\right)^{2} \cap D\right)$.

$$
\begin{gathered}
E\left((N \times N)\left(\left[m_{1}, m_{1}+1\right)^{2} \cap D\right)\right)=E\left(X \mid \Omega^{\prime}\right) P\left(\Omega^{\prime}\right)+E\left(X \mid \Omega^{\prime \prime}\right) P\left(\Omega^{\prime \prime}\right)= \\
=E\left(X \mid \Omega^{\prime}\right)= \\
=E\left(X \mid \Omega^{\prime} \cap \Omega_{2, m_{1}}\right) P\left(\Omega_{2, m_{1}}\right)+E\left(X \mid \Omega^{\prime} \cap\left(\Omega^{\prime} \backslash \Omega_{2, m_{1}}\right)\right) P\left(\Omega^{\prime} \backslash \Omega_{2, m_{1}}\right) .
\end{gathered}
$$

Using Theorem's 4.3 notation we write

$$
\begin{array}{r}
X(\omega)=\sum n_{k}^{2}(\omega)=\sum n_{k}(\omega)=N_{\omega}\left[m_{1}, m_{1}+1\right) \text { for all } \omega \in \Omega^{\prime} \backslash \Omega_{2, m_{1}}, \\
\text { and } X(\omega)=\sum n_{k}^{2}(\omega) \geq 3+\sum n_{k}(\omega)=3+N_{\omega}\left[m_{1}, m_{1}+1\right), \\
\text { for all } \omega \in \Omega^{\prime} \cap \Omega_{2, m_{1}} .
\end{array}
$$

Thus, since $P\left(\Omega^{\prime}\right)=1$ and $N$ satisfies hypothesis *A,

$$
\begin{gathered}
E\left((N \times N)\left(\left[m_{1}, m_{1}+1\right)^{2} \cap D\right)\right) \geq \\
\geq\left(3+E\left(N\left[m_{1}, m_{1}+1\right) \mid \Omega_{2, m_{1}}\right)\right) P\left(\Omega_{2, m_{1}}\right)+ \\
+E\left(N\left[m_{1}, m_{1}+1\right) \mid \Omega^{\prime} \backslash \Omega_{2, m_{1}}\right) P\left(\Omega^{\prime} \backslash \Omega_{2, m_{1}}\right) \\
\left.=E N \pi_{1}\left(\left[m_{1}, m_{1}+1\right)^{2} \cap D\right)\right)+3 P\left(\Omega_{2, m_{1}}\right)> \\
\left.>E N \pi_{1}\left(\left[m_{1}, m_{1}+1\right)^{2} \cap D\right)\right),
\end{gathered}
$$

and this inequality implies that $N$ is not under hypothesis ${ }^{*} \mathrm{~A}$.

Theorem 4.6. A point process is under hypothesis ${ }^{*} A$ if and only if it is simple and it satisfies hypothesis ${ }^{*} B$.

Proof: If part: Corollary 4.1.

Only if part: observe that ${ }^{*} \mathrm{~A}$ implies ${ }^{*} \mathrm{~B}$ by definition and that ${ }^{*} \mathrm{~A}$ implies simpleness by Theorem 4.5.

Lemma 4.3. Let $a$ be a real number and $\delta:[a, \infty) \subset \mathbb{R} \rightarrow \mathbb{R}_{+}^{*}$ be $a$ positive function. Then from the cover of $[a, \infty)$, given by $\{[t, t+\delta(t)) \mid t \in$ $[a, \infty)\}=\mathcal{C}$, we can choose for all $x>a$ an enumerable disjoint subclass $\left\{\left[t_{i}, t_{i}+\delta\left(t_{i}\right)\right) \mid t_{i} \in I\right\}$ of $\mathcal{C}$ that covers $[a, x)$.

Proof: Let $\mathcal{O}$ be a non-enumerable ordinal and form the function $f$ : $\mathcal{O} \rightarrow \overline{\mathbb{R}}$ such that $f(0)=a$ and, for all $c \in \mathcal{O}, f\left(c^{+}\right)=f(c)+\delta(f(c))$, where $c^{+}$is the successor of $c$. In case $l \in \mathcal{O}$ is a limit ordinal then $f(l)=$ $\sup \{f(c) \mid c<l\}$. This is a strictly increasing function in $f^{-1}(\mathbb{R})$. Take the interval $\bigcup_{c \in \mathcal{O}}\left[f(c), f\left(c^{+}\right)\right)$. This interval is formed by a disjoint union of intervals and its length is given by $\sum_{c \in \mathcal{O}}\left(f\left(c^{+}\right)-f(c)\right)=\sum_{c \in \mathcal{O}}(\delta(f(c))$. 
Since card $\mathcal{O}>$ card $\mathbb{N}$ and $\delta(f(c))>0$ for all $c \in \mathcal{O}$ we have

$$
\sum_{c \in \mathcal{O}}(\delta(f(c))=\infty
$$

which implies that $\bigcup_{c \in \mathcal{O}}\left[f(c), f\left(c^{+}\right)\right) \supset[a, \infty)$.

Now, let $x_{*}=\inf \left\{x \mid[a, x)\right.$ can not be covered by $\bigcup_{c \in \mathcal{O}_{o}}\left[f(c), f\left(c^{+}\right)\right)$, $\mathcal{O}_{o}$ enumerable ordinal $\}$. Clearly $x_{*} \geq a+\delta(a)>a$.

Suppose $x_{*} \in \mathbb{R}$. In this way, for all $y<x_{*}$ there exists an enumerable ordinal $\mathcal{O}_{o, y}$ such that $[a, y) \subset \bigcup_{c \in \mathcal{O}_{o, y}}\left[f(c), f\left(c^{+}\right)\right)$.

Take, for all $n \in \mathbb{N}^{*}, y_{n}=x_{*}-\left(x_{*}-a\right) /(n+1)$. Thus, the interval $\left[a, x_{*}\right)$ is contained in the union $\bigcup_{n \in \mathbb{N}^{*}}\left(\bigcup_{c \in \mathcal{O}_{o, y_{n}}}\left[f(c), f\left(c^{+}\right)\right)\right)$that is itself an enumerable union and can be written as $\bigcup_{c \in \mathcal{O}_{o}^{*}}\left[f(c), f\left(c^{+}\right)\right), \mathcal{O}_{o}^{*}$ enumerable ordinal. Now, as a direct consequence of the definition of $x_{*}$, the interval $\left[a, x_{*}+\delta\left(x_{*}\right)\right)$ can not be covered by any enumerable union of the form $\bigcup_{c \in \mathcal{O}_{o}}\left[f(c), f\left(c^{+}\right)\right)$, and, at the same time, $\left[a, x_{*}+\delta\left(x_{*}\right)\right)=$ $\left(\bigcup_{c \in \mathcal{O}_{o}^{*}}\left[f(c), f\left(c^{+}\right)\right)\right) \cup\left[x_{*}, x_{*}+\delta\left(x_{*}\right)\right)=\bigcup_{c \in\left(\mathcal{O}_{o}^{*}\right)^{+}}\left[f(c), f\left(c^{+}\right)\right)$, that is, it is covered by an enumerable union of that very form.

The contradiction above leads us to the conclusion that $x_{*} \notin \mathbb{R}$ and that for all $x$ there exists an enumerable disjoint subclass of $\mathcal{C}$ that covers $[a, x)$.

Theorem 4.7. If $N$ is a point process under hypothesis ${ }^{*} B$ then it is simple.

Proof: Since $N$ in under hypothesis ${ }^{*} \mathrm{~B}$ we can write $\forall \Delta \subset \mathbb{R}, \Delta$ interval, $\forall t \in \mathbb{R} E N(\Delta)=P\{N(\Delta)=1\}+o_{t, \Delta}(|\Delta|)$. Thus, $\sum_{n \geq 2} P\{N(\Delta)=n\}=$ $P\{N(\Delta)>1\} \leq o_{t, \Delta}(|\Delta|)$. Let $\Omega_{2}=\left\{\omega \in \Omega \mid \exists t \in \mathbb{R}^{-2} N_{\omega}(\{t\})>1\right\}$ and $\Omega_{2, m}=\left\{\omega \in \Omega \mid \exists t \in[m, m+1) N_{\omega}(\{t\})>1\right\}$. We have $\Omega_{2}=\bigcup_{m \in \mathbb{Z}} \Omega_{2, m}$ and $P\left(\Omega_{2}\right) \leq \sum_{m \in \mathbb{Z}} P\left(\Omega_{2, m}\right)$.

For every $\left\{\Delta_{i}^{(m)}\right\}_{i \in I_{m}}$ disjoint enumerable cover of $[m, m+1)$ formed by intervals $\Delta_{i}^{(m)}$ contained in $[m, m+1)$, if $\Omega_{2, m, i}=\left\{\omega \in \Omega \mid \exists t \in \Delta_{i}^{(m)} N_{\omega}(\{t\})\right.$ $>1\}$ then we have $\Omega_{2, m}=\bigcup_{i \in I_{m}} \Omega_{2, m, i}$ and $P\left(\Omega_{2, m}\right) \leq \sum_{i \in I_{m}} P\left(\Omega_{2, m, i}\right)$.

Now $\forall t \in \mathbb{R} \forall \varepsilon>0 \exists \delta(t, \varepsilon)>0(0<|\Delta|<\delta(t, \varepsilon)) \rightarrow \frac{o_{t, \Delta}(|\Delta|)}{|\Delta|}<\varepsilon$. This implies that $\forall t \in \mathbb{R} \forall \varepsilon>0 P\{N([t, t+\delta(t, \varepsilon) / 2)>1\}<\varepsilon \delta(t, \varepsilon) / 2$.

Form a cover of $[m, m+1)$ of the form $\{[t, t+\delta(t, \varepsilon) / 2) \cap[m, m+1) \mid t \in$ $[m, \infty)\}$. From this non-enumerable cover, as a consequence of Lemma 4.3 , we can choose a disjoint enumerable sub-cover of $[m, m+1),\left\{\Delta_{i}^{(m)}=\right.$ $\left.\left[t_{i}, t_{i}+\delta\left(t_{i}, \varepsilon\right) / 2\right) \cap[m, m+1) \mid i \in I_{m}\right\}$, for some $I_{m}$ enumerable set. 
Observe that $\left.\Omega_{2, m, i} \subset\left\{\omega \in \Omega \mid N_{(} \omega\right)\left(\Delta_{i}^{(m)}\right)>1\right\}$. So we can write: $P\left(\Omega_{2, m}\right) \leq \sum_{i \in I_{m}} P\left\{N\left(\Delta_{i}^{(m)}\right)>1\right\} \leq \sum_{i \in I_{m}} \varepsilon \delta\left(t_{i}, \varepsilon\right) / 2=\varepsilon$.

Now, letting $\varepsilon \rightarrow 0$ we have $P\left(\Omega_{2, m}\right)=0$ which implies $P\left(\Omega_{2}\right)=0$. This proves that $N$ is simple.

Theorem 4.8. Equivalence Theorem for Point Processes on the Real Line. A point processes $N$ is under hypothesis ${ }^{*} B$ if and only if it is under hypothesis ${ }^{*} A$.

Proof: By Theorem 4.6 hypothesis $* \mathrm{~A}$ is equivalent to hypothesis $* \mathrm{~B}$ and simpleness. By Theorem 4.7, hypothesis ${ }^{*} \mathrm{~B}$ and simpleness is equivalent to hypothesis *B.

Theorem 4.9. Chain of class inclusions Let $S$ be the class of simple point processes on $\mathbb{R}$. Then we have the following inclusions: $\mp \varsubsetneqq B \varsubsetneqq * A=$ $* B \varsubsetneqq S$.

Proof: By definition, $A \subset B$. Theorem 4.1 guarantees $B \subset * B$ and Theorem 4.8 implies $* B=* A$. Now, $* A \subset S$ by Theorem 4.5. Thus $A \subset B \subset * B=* A \subset S$.

Let $N_{1}$ be the independent sum of an homogeneous Poisson process with a deterministic point process whose only occurrence is at $t=0$ with probability one. This is a simple point process with a fixed atom at 0 . Thus $E N_{1}$ is not absolutely continuous w.r.t the Lebesgue measure, and, consequently, $N_{1} \notin * B$.

Take $N_{2}$ a non homogeneous Poisson process with intensity equals to 1 on $(-\infty, 0)$ and intensity equals to 2 on $[0, \infty)$. Clearly, $N_{2} \in * B$. Since its intensity is not continuous, $N_{2}$ does not belong to $B$.

Take $N_{3}$ the point process defined by the following procedure: Let $X$ : $\Omega \rightarrow[0,1)$ be an uniform random variable and define the trajectories of $N_{3}$ by $N_{3, \omega}=X(\omega)+\mathbb{Z}$. Taking $\delta=0.1$ and $K_{\delta}=1$, for example, we have, for all $\Delta$ with length less than $\delta$, that $P\left(N_{3}(\Delta)=n\right)=0 \leq K_{\delta}|\Delta|^{n}$ for all $n>1$. We also have

$$
\lim _{|\Delta| \rightarrow 0, t \in \Delta} \frac{1}{|\Delta|} P\left\{N_{3}(\Delta)=1\right\}=\lim _{|\Delta| \rightarrow 0, t \in \Delta} \frac{|\Delta|}{|\Delta|}=1,
$$

uniformly in $t$. Thus, $N_{3} \in B$. Now, choosing $\Delta_{1}=[t, t+h)$ and $\Delta_{2}=$ $[t+1, t+1+h)$ we have

$p_{2}(t, t+1)=\lim _{(h, h) \rightarrow 0} \frac{1}{h^{2}} P\left\{N\left(\Delta_{1}\right)=1, N\left(\Delta_{2}\right)=1\right\}=\lim _{(h, h) \rightarrow 0} \frac{h}{h^{2}}=\infty$, 
and $N_{3} \notin * A$. This completes the proof.

\section{Equivalence Under Poisson Hypothesis}

We recall that one important point process is the (non-homogeneous) Poisson process, for which we are given a non-decreasing, right-continuous function $\Lambda(t)$, such that whenever $\left(\alpha_{i}, \beta_{i}\right] \cap\left(\alpha_{j}, \beta_{j}\right]=\emptyset$, for all $i \neq j$,

$P\left(N\left(\alpha_{1}, \beta_{1}\right]=n_{1}, \ldots, N\left(\alpha_{k}, \beta_{k}\right]=n_{k}\right)=\prod_{j=1}^{k}\left(\frac{\left[\Lambda\left(\beta_{j}\right)-\Lambda\left(\alpha_{j}\right)\right]^{n_{j}}}{n_{j} !} e^{-\left[\Lambda\left(\beta_{j}\right)-\Lambda\left(\alpha_{j}\right)\right]}\right)$.

As a consequence of this formula, the random variables $N\left(\alpha_{j}, \beta_{j}\right]$ are Poisson distributed with mean $\Lambda\left(\beta_{j}\right)-\Lambda\left(\alpha_{j}\right)$ and form a completely independent set. Equivalently, the number of events in any collection of disjoint intervals are independent and Poisson distributed. An important special case is when $\Lambda(t)=\lambda t, \lambda$ being the mean intensity of the process.

Observe that a Poisson process under hypothesis ${ }^{*} \mathrm{~B}$ has no fixed atoms otherwise we wouldn't have $E N \ll \ell$. Note also that a Poisson processes has no fixed atoms if and only if $\Lambda$ is continuous.

The following lemma generalizes Lemma 4.2

Lemma 5.1. If for a point process $N$ there are functions $K: \mathbb{R} \rightarrow \mathbb{R}_{+}^{*}$, bounded over bounded sets, and $\delta: \mathbb{R} \rightarrow \mathbb{R}_{+}^{*}$ with the property that in $f_{x \in A}$ $\delta(x)>0$ for all bounded sets $A \subset \mathbb{R}$, such that for all intervals $\Delta$ of the form $[t, t+|\Delta|)$ with length $|\Delta|<\delta(t)$, all integers $n>1$ and all $t \in \mathbb{R}$ we have $P\{N(\Delta)=n\} \leq K(t)|\Delta|^{n}$, then $N$ is simple.

Proof: $\quad$ Let $a, b \in \mathbb{R}, a<b, \Delta_{i}=\left[a+\frac{i(b-a)}{n}, a+\frac{(i+1)(b-a)}{n}\right), K_{a, b}=$ $\sup \{K(t) \mid t \in[a, b)\}$ and $\delta_{a, b}=\inf \{\delta(t) \mid t \in[a, b)\}$. There is an $n^{*}$ such that $\frac{b-a}{n^{*}}<\min \left\{1, \delta_{a, b}\right\}$. For all $n>n^{*}$ we have

$$
\begin{gathered}
P\{\omega \in \Omega \mid \exists t \in[a, b) N(\{t\})>1\} \leq P\left\{\omega \in \Omega \mid \exists i, 0 \leq i \leq n-1, N\left(\Delta_{i}\right)>1\right\} \\
\leq \sum_{i=1}^{n} P\left\{N\left(\Delta_{i}\right)>1\right\} \leq \sum_{i=1}^{n}\left(\sum_{j \geq 2} K_{a, b}\left|\Delta_{i}\right|^{j}\right)= \\
=K_{a, b}\left(\frac{b-a}{n}\right)\left(\frac{1}{1-(b-a) / n}\right) \sum_{i=1}^{n} \frac{b-a}{n}=\frac{K_{a, b}(b-a)^{2}}{n-(b-a)} \rightarrow 0 \text { as } n \rightarrow \infty .
\end{gathered}
$$


In this way,

$$
\begin{gathered}
0 \leq P\{\omega \in \Omega \mid \exists t \in \mathbb{R} N(\{t\})>1\} \leq \\
\leq \sum_{m \in \mathbb{Z}} P\{\omega \in \Omega \mid \exists t \in[m, m+1) N(\{t\})>1\} \leq \\
\leq \sum_{m \in \mathbb{Z}} 0=0 .
\end{gathered}
$$

We already know that classes $* A$ and $* B$ are equivalent for point processes on $\mathbb{R}$. The following theorem is weaker than Theorem 4.8 since we assume that $N$ is a Poisson process with $p_{N} \in \mathcal{L}^{1}$. As a matter of fact it is presented here because its proof, as well as the previous lemma, are suitable for generalizations to higher dimensions.

Theorem 5.1. If $N$ is a Poisson process with $p_{N} \in \mathcal{L}^{1}$ then $N$ is under hypothesis ${ }^{*} B$ if and only if $N$ is under hypothesis ${ }^{*} A$.

Proof: It suffices to prove that $N$ is simple. Observe that $\forall x \geq 0$ $\max _{n \in \mathbb{N}}\left\{\frac{x^{n}}{n !}\right\} \leq x^{\lfloor x\rfloor}$ and also that $K_{1}: \mathbb{R} \rightarrow \mathbb{R}, K_{1}(x)=x^{\lfloor x\rfloor}$ for positive $x$ and $K_{1}(x)=0$ otherwise, is a monotone non-decreasing function that belong to $\mathcal{L}^{1}$. Construct the function

$$
K(t)=\left\{\begin{array}{l}
K_{1}\left(\sup _{[-2,2]} p_{N}\right), \text { for } t \in[-1,1] \\
K_{1}\left(\sup _{[-3,3]} p_{N}\right), \text { for } t \in[-2,2] \backslash[-1,1], \\
\ldots \\
K_{1}\left(\sup _{[-(m+1),(m+1)]} p_{N}\right), \text { for } t \in[-m, m] \backslash[-(m-1),(m-1)], \\
\ldots
\end{array}\right.
$$

Clearly we have $K \in \mathcal{L}^{1}$.

Since $N$ satisfies hypothesis ${ }^{*} \mathrm{~B}$ we have:

$$
E N([a, b))=\int_{a}^{b} p_{N}(t) \mathrm{d} t \leq(b-a) \sup _{t \in[a, b]} p_{N}(t)
$$

from which $\left(\frac{\Lambda(b)-\Lambda(a)}{b-a}\right) \leq \sup _{t \in[a, b]} p_{N}(t)$ 
Now, take $\delta: \mathbb{R} \rightarrow \mathbb{R}$ the constant function $\delta(t)=1 / 2$. For all intervals $\Delta=[a, b)$ with length $|\Delta|<\delta(t)=1 / 2$ we have

$$
\begin{aligned}
P\{N[a, b)=n\} & =\frac{1}{n !}\left(\frac{\Lambda(b)-\Lambda(a)}{b-a}\right)^{n} e^{-(\Lambda(b)-\Lambda(a))}(b-a)^{n} \\
& \leq\left(\frac{\Lambda(b)-\Lambda(a)}{b-a}\right)^{\left\lfloor\frac{\Lambda(b)-\Lambda(a)}{b-a}\right\rfloor}(b-a)^{n} \\
& \leq\left(\sup _{\left[a, a+\frac{1}{2}\right)} p_{N}(t)\right)^{\left\lfloor a, a+\frac{1}{2}\right)} p_{N}(b-a)^{n} \leq K(a)(b-a)^{n},
\end{aligned}
$$

and Lemma's 5.1 hypothesis is fulfilled.

\section{Conclusions}

In this work we have defined four classes of point processes. We have focused on point processes on the real line but the definitions of hypothesis and classes are suitable for direct generalization to point process on $\mathbb{R}^{m}$ and more general spaces. An important result is the equivalence of classes ${ }^{*} \mathrm{~B}$ and ${ }^{*} \mathrm{~A}$ for point process on the real line. We observe that to obtain this equality of classes we have strongly used the order of the real numbers, although for Poisson process such that $p_{N} \in \mathcal{L}_{1}$, this equivalence was derived in such way that generalization for higher dimensions are possible.

\section{References}

1. Brillinger, D.R. (1975). Statistical inference for stationary point processes. Stochastic Processes and Related Topics, 1, 55-99.

2. Brillinger, D.R. (1978). Comparative aspects of the study of ordinary time series and point processes. Developments in Statistics, 1 , 33-133.

3. Brillinger, D.R. (1997). Some wavelet analyses of point processes data. The Third First Asilomar Conference on Signals, Systems and Computers. IEEE Computer Society, 1087-1091.

4. de Miranda, J.C.S. (2003). Sobre a estimação da intensidade dos processos pontuais via ondaletas. Tese de Doutorado IME-USP.

5. de Miranda, J.C.S. Characterization of the Intensity for a Class of Point Processes. Technical Report Institute of Mathematics and Statistics - RT MAT 2005-13.

6. de Miranda, J.C.S. Neglecting Higher Order Infinitesimals and the Study of Some Measures. Technical Report Institute of Mathematics and Statistics- RT MAT 2005-14. 
7. de Miranda, J.C.S. Some Wide Classes of Point Processes - Interrelationships and Three Working Properties. Technical Report Institute of Mathematics and Statistics RT MAT 2005-15.

8. de Miranda, J.C.S. Non Internally Correlated Point Processes. RT MAE 2009-06.

9. de Miranda, J.C.S. and Morettin, P.A. On the Estimation of the Intensity of Point Processes via Wavelets. Technical Report Institute of Mathematics and Statistics - RT MAE 2006-06.

10. de Miranda, J.C.S. and Morettin, P.A. Estimation of the Density of Point Processes on $\mathbb{R}^{m}$ via Wavelets. Technical Report Institute of Mathematics and StatisticsRT MAT 2005-09.

11. de Miranda, J.C.S ; Morettin, P.A. Estimation of the Intensity of Non-homogeneous Point Processes via Wavelets. Annals of the Institute of Statistical Mathematics. Vol 63, no 6, pages 1221-1246, 2011. 